\title{
Real-world experience of erenumab in patients with chronic or episodic migraine in the UAE
}

Taoufik Alsaadi ( $\square$ t.alsaadi@americancenteruae.com )

Department of Neurology, American Center of Psychiatry and Neurology, Abu Dhabi

\section{Suzan Noori}

University of Sharjah, Sharjah

\section{Razmig Varakian}

Novartis Middle East FZE, Representative Office, Dubai

\section{Saly Youssef}

Novartis Middle East FZE, Representative Office, Dubai

Abu Baker Almadani

Neurology Department, Rashid Hospital, Dubai

\section{Research Article}

Keywords: Erenumab, calcitonin gene-related peptide monoclonal antibody, migraine, safety, real-world evidence, UAE, CGRP

Posted Date: February 16th, 2022

DOI: https://doi.org/10.21203/rs.3.rs-1305232/v1

License: (c) (i) This work is licensed under a Creative Commons Attribution 4.0 International License. Read Full License 


\section{Abstract}

Background: Erenumab is a fully human monoclonal antibody and a highly potent, first-in-class calcitonin gene-related peptide receptor inhibitor approved for migraine prevention in adults. Randomised, placebocontrolled trials show that erenumab treatment results in clinically meaningful responses, including significant reductions in monthly migraine days. Real-world evidence of the effectiveness of erenumab in patients with migraine is accruing, but gaps remain, and findings may vary according to region. We evaluated the usage patterns and effectiveness of erenumab in real-world settings in patients with migraine in the United Arab Emirates (UAE).

Methods: This retrospective, observational real-world study enrolled patients $\geq 18$ years with migraine who were prescribed erenumab in the UAE. Data were collected at baseline and Months 1, 3 and 6 . The primary study objective was to characterise usage patterns of erenumab in patients with chronic migraine (CM) or episodic migraine (EM) in real-world settings in the UAE.

Results: Of the 166 patients, 124 (74.7\%) were females. The mean (standard deviation) age at migraine onset was 29 (7.93) years. Seventy-one patients (42.8\%) had CM and 95 (57.2\%) had EM.

In the overall population, the mean monthly headache/migraine days (MHD) at baseline was 15.7 (8.45) and mean change from baseline was -8.2 (8.83) at Month 1, -11.0 (9.15) at Month 3 and -11.3 (8.90) at Month 6 . The mean change from baseline in monthly acute migraine-specific medication days (MSMD) was -9.0 (8.07) at Month 1, -9.7 (8.73) at Month 3 and -10.7 (8.95) at Month 6. At all time points, most patients achieved at least 50\% reduction in MHD (80\%-91\%) and MSMD (84\%-94\%).

Similar reductions in MHD and MSMD and clinical benefit in CM or EM were seen with erenumab monotherapy or erenumab add-on therapy, with or without dose escalation and for treatment naïve or $\geq 1$ previous preventive treatment failures, with additional clinical benefit in the erenumab add-on therapy and dose escalation to $140 \mathrm{mg}$ subgroups.

Conclusion: In this real-world study on erenumab use in the UAE, patients prescribed erenumab achieved clinically meaningful reductions in MHD and MSMD at all assessed time points. Erenumab was well tolerated with no new safety events.

Trial registration: Not applicable

\section{Background}

Migraine is a common chronic neurovascular disease associated with a significant personal burden of pain, disability, and reduced quality of life. The personal, social, and healthcare-related economic burden associated with the disease is also significant $[1,2]$. Migraine remains under-recognised and undertreated [1]. Despite being under-recognised, the Global Burden of Disease 2016 study suggests a global age-standardised migraine prevalence of $14.3 \%$ [3]. The disease represents the second-largest 
cause of disability globally, with women having a higher number of years lived with disability (YLD) than men [4].

Headache disorders are the third-highest cause of YLD in the Middle East [4]. The prevalence of migraine in Arab countries is similar to that estimated worldwide but varies by region. The prevalence of migraine is reported to be $2.6 \%-5 \%$ in Saudi Arabia and $7.9 \%$ in Qatar, whereas the 1-year migraine prevalence in Oman is reported to be $10.1 \%$ [5] and in Kuwait is reported to be $23 \%$ [6].

Preventive treatment of migraine is typically based on pharmacological therapies such as topiramate, valproate, $\beta$-blockers and amitriptyline, which were not developed specifically for migraine management. These non-specific therapies have been associated with high levels of discontinuation owing to a lack of efficacy and/or poor tolerability [7]. Calcitonin gene-related peptide (CGRP) plays a key role in migraine pathophysiology [8]; hence, the CGRP receptor is a relevant target for the preventive treatment of migraine $[9,10]$.

Erenumab, a fully human immunoglobulin $\mathrm{G} 2$ monoclonal antibody ( $\mathrm{mAb}$ ), is a highly potent and selective antagonist of the canonical CGRP receptor [11]. Erenumab was specifically developed to treat migraine [11] and is a first-in-class mAb approved by the United States Food and Drug Administration (May 2018) and the European Medicines Agency (July 2018) for migraine prevention in adults [12, 13]. Erenumab has since been approved in several other countries, including the United Arab Emirates (UAE), and is administered as monthly subcutaneous injections at a dose of either $70 \mathrm{mg}$ or $140 \mathrm{mg}$ [12]. In the $\mathrm{UAE}$, the recommended starting dose is $70 \mathrm{mg}$, which can be escalated to $140 \mathrm{mg}$ if required.

Erenumab has been previously shown to reduce the monthly migraine days (MMD) and increase the likelihood of achieving a clinically meaningful response ( $\geq 50 \%$ reduction from baseline in MMD) at all monthly assessment points tested in placebo-controlled trials, including pivotal phase $2 / 3$ trials of episodic migraine (EM) [14-16] and chronic migraine (CM) [17]. There is a need to understand the regionspecific usage patterns and effectiveness of erenumab. This study aimed to characterise the realworld usage patterns and effectiveness of erenumab in the UAE.

\section{Methods}

\section{Study design}

This was a retrospective, non-interventional, observational, real-world study that enrolled patients diagnosed with either EM (up to 14 monthly headache/migraine days [MHD] [18]) or CM ( $\geq 15 \mathrm{MHD}$, of which at least 8 had to be typical migraine days), including patients with medication overuse headache $(\mathrm{MOH})$ treated with erenumab at one of the four sites across three medical centres in the UAE. MOH was defined according to the International Classification of Headache Disorders, $3^{\text {rd }}$ edition guidelines [19].

Patients aged $\geq 18$ years at the time of erenumab treatment initiation who had received at least three doses of erenumab before entering the study were included in the analysis. Patients aged $\geq 50$ years at 
migraine onset were excluded from this analysis. Patients who were previously treatment naïve (i.e., for whom erenumab was prescribed as the first preventive therapy) and those who had $\geq 1$ previous preventive treatment failure (PPTF) were included. were included. Patients for whom erenumab was prescribed as the first preventive therapy had to have received at least 3 doses of erenumab before entering the study. For patients entering the study on erenumab $70 \mathrm{mg}$, the dose could be escalated to $140 \mathrm{mg}$ after an initial three-month administration of $70 \mathrm{mg}$ upon the treating physician's decision.

Demographics, baseline information, clinical characteristics and treatment history were extracted from the medical records of eligible patients. Data were collected at baseline, and at Months 1, 3 and 6 , per availability in the medical records. Patient records with at least 6 months of follow-up from the time of erenumab treatment initiation were included in the analysis. The collected data are presented by migraine type (EM or CM); by erenumab monotherapy (with or without a sufficient washout period from the previous preventive treatments) or erenumab add-on therapy; by patients who entered the study and remained on erenumab $70 \mathrm{mg}$ or those who were prescribed erenumab at the 70 -mg dose and were subsequently escalated to erenumab $140 \mathrm{mg}$; and by treatment naïve or with PPTFs.

All relevant ethical, health authority, data privacy and site-specific approvals were obtained prior to the start of the study. The study was conducted in accordance with the principles of the Declaration of Helsinki. Informed consent was obtained from patients before data collection. Depending on each site's local Institutional Review Board (IRB) regulations, a waiver of informed consent from the corresponding ethics committee was obtained. All authors were given access to the study data.

\section{Objectives and endpoints}

The primary objective of the study was to characterise the usage patterns of erenumab in patients with $\mathrm{EM}$ or $\mathrm{CM}$ starting treatment with erenumab in a real-world setting in the UAE. The secondary objectives of the study were to evaluate the change in MHD in patients with $\mathrm{CM}$ and $\mathrm{EM}$ and the change in monthly acute migraine-specific medication days (MSMD), and to summarise patient demographic characteristics and disease/medication history.

Exploratory objectives included a description of compliance and adherence to erenumab, the response rate in terms of reduction in MHD across the study period and the incremental benefit of dose escalation from $70 \mathrm{mg}$ to $140 \mathrm{mg}$. The enrolled analysis set included all patients who signed an informed consent form (ICF) or a waiver of ICF (wherever applicable) and met the inclusion and exclusion criteria. Male and female patients $\geq 18$ years at the time of erenumab treatment initiation and diagnosis of EM or CM, including those with $\mathrm{MOH}$ and those treated with at least three doses of erenumab were enrolled. Patients $>50$ years of age at migraine onset were excluded. The full analysis set (FAS) included all enrolled patients who received at least one dose of the study drug and had at least one evaluable post-baseline assessment. The safety analysis set included all patients who received at least one dose of the study drug. 
Current disease status, including the duration of disease, the number of MHD, previous and current preventive treatments and comorbidities (anxiety, depression, diabetes, or obesity) were assessed at baseline. Medical history/current medical conditions and adverse events (AEs) were coded using the Medical Dictionary for Regulatory Activities.

\section{Statistical analysis}

Sample size calculations indicated that a minimum of 160 patients were required to characterise the effectiveness of erenumab in patients with CM or EM. Given the exploratory nature of the endpoints, descriptive statistics are presented. No formal hypotheses were defined for the present exploratory analyses; therefore, no inferential testing was performed on any endpoint. Categorical data are reported as frequencies and percentages, and continuous data as mean, standard deviation (SD), median, minimum, and maximum. P-values were derived from Chi-Square test or exact test for categorical variables and from t-test or Wilcoxon test for continuous variables. Owing to the descriptive and observatory nature of this study, missing data were not imputed.

\section{Results}

\section{Demographics and baseline characteristics}

Overall, 166 patients were enrolled and completed the study; 135 patients (81.3\%) completed the study per protocol from baseline to Month 6 , and 31 patients (18.7\%) completed the study protocol partially. Of the 166 patients, a majority ( $n=124,74.7 \%)$ were female (Table 1). Seventy-one (42.8\%) patients had CM and $95(57.2 \%)$ had EM.

The demographic and baseline characteristics by $\mathrm{CM}$ and EM are presented in Table 1. The overall mean (SD) age of patients was 37.8 (8.99) years (Table 1). The difference in mean age between patients with $\mathrm{CM}$ and EM was not statistically significant; however, for age categories, the difference was statistically significant $(p=0.0498)$. Most patients were aged $\geq 35$ years to $<50$ years $(53.6 \%)$ and a similar proportion of those with $\mathrm{CM}$ and EM were in this category. A higher proportion of patients with EM tended to be in the $\geq 18$ to $<35$-year category and a lower proportion in the $\geq 50$-year category compared with those with CM (EM: $43.2 \%$ and $3.2 \%$; CM: $33.8 \%$ and $12.7 \%$, respectively). A family history of migraine was reported for $30.7 \%$ of patients (Table 1 ). The mean (SD) age at onset of migraine for the overall study population was 29.0 (7.93) years, 29.4 (8.35) years for CM and 28.7 (7.63) years for EM (Table 1).

\section{Table 1}

Patient demographics and baseline characteristics 


\begin{tabular}{|c|c|c|c|c|}
\hline & $\begin{array}{l}\text { Overall } \\
\mathrm{N}=166\end{array}$ & $\begin{array}{l}\text { Chronic } \\
\text { migraine } \\
\mathrm{N}=71\end{array}$ & $\begin{array}{l}\text { Episodic } \\
\text { migraine } \\
\mathrm{N}=95\end{array}$ & p-value \\
\hline Age, years, mean (SD) & $\begin{array}{l}37.8 \\
(8.99)\end{array}$ & $\begin{array}{l}38.8 \\
(9.51)\end{array}$ & $\begin{array}{l}37.1 \\
(8.57)\end{array}$ & 0.2502 \\
\hline \multicolumn{5}{|l|}{ Age categories, years, n (\%) } \\
\hline$\geq 18$ to $<35$ & $65(39.2)$ & $24(33.8)$ & $41(43.2)$ & 0.0498 \\
\hline$\geq 35$ to $<50$ & $89(53.6)$ & $38(53.5)$ & $51(53.7)$ & \\
\hline$\geq 50$ & $12(7.2)$ & $9(12.7)$ & $3(3.2)$ & \\
\hline Sex, female, n (\%) & $\begin{array}{l}124 \\
(74.7)\end{array}$ & $49(69.0)$ & 75 (78.9) & \\
\hline \multicolumn{5}{|l|}{ Ethnicity, n (\%) } \\
\hline Local & $\begin{array}{l}123 \\
(74.1)\end{array}$ & $53(74.6)$ & $70(73.7)$ & 0.8885 \\
\hline Non local & $43(25.9)$ & $18(25.4)$ & $25(26.3)$ & \\
\hline Arab & $27(16.3)$ & $12(16.9)$ & $15(15.8)$ & \\
\hline Asian & $4(2.4)$ & $1(1.4)$ & $3(3.2)$ & \\
\hline Western & $4(2.4)$ & $2(2.8)$ & $2(2.1)$ & \\
\hline European & $7(4.2)$ & $3(4.2)$ & $4(4.2)$ & \\
\hline Other & $1(0.6)$ & 0 & $1(1.1)$ & \\
\hline $\mathrm{BMI}, \mathrm{kg} / \mathrm{m}^{2}$, mean $(\mathrm{SD})$ & $\begin{array}{l}28.2 \\
(4.76)\end{array}$ & $\begin{array}{l}28.4 \\
(4.97)\end{array}$ & $\begin{array}{l}28.1 \\
(4.66)\end{array}$ & 0.8322 \\
\hline \multicolumn{5}{|l|}{ BMI category, $\mathrm{kg} / \mathrm{m}^{2}, \mathrm{n}(\%)$} \\
\hline$\geq 18.5$ to $<25$ & $20(26.0)$ & $8(25.8)$ & $12(26.1)$ & 0.9985 \\
\hline$\geq 25$ to $<30$ & $32(41.6)$ & $13(41.9)$ & $19(41.3)$ & \\
\hline$>30$ & $25(32.5)$ & $10(32.3)$ & $15(32.6)$ & \\
\hline \multicolumn{5}{|l|}{ Family history of migraine, $\mathrm{n}(\%)$} \\
\hline Yes & $51(30.7)$ & $21(29.6)$ & $30(31.6)$ & 0.8952 \\
\hline No & $76(45.8)$ & $34(47.9)$ & $42(44.2)$ & \\
\hline Unknown & $39(23.5)$ & $16(22.5)$ & $23(24.2)$ & \\
\hline Duration of migraine, years, mean (SD) & $5.0(9.75)$ & $\begin{array}{l}6.0 \\
(7.82)\end{array}$ & $\begin{array}{l}4.4 \\
(10.92)\end{array}$ & 0.0223 \\
\hline Age at migraine onset, years, mean (SD) & 29.0 & 29.4 & 28.7 & 0.5352 \\
\hline
\end{tabular}




\begin{tabular}{|c|c|c|c|c|}
\hline & $(7.93)$ & $(8.35)$ & $(7.63)$ & \\
\hline MHD over the past 1 month, days, mean (SD) & $\begin{array}{l}15.6 \\
(8.54)\end{array}$ & $\begin{array}{l}22.9 \\
(6.35)\end{array}$ & $\begin{array}{l}9.0 \\
(3.05)\end{array}$ & $<0.0001$ \\
\hline MHD at baseline, days, mean (SD) & $\begin{array}{l}15.7 \\
(8.45)\end{array}$ & $\begin{array}{l}22.9 \\
(6.57)\end{array}$ & $\begin{array}{l}9.2 \\
(2.93)\end{array}$ & NA \\
\hline MSMD at baseline, days, mean (SD) & $\begin{array}{l}12.8 \\
(10.00)\end{array}$ & $\begin{array}{l}18.6 \\
(8.55)\end{array}$ & $\begin{array}{l}7.4 \\
(8.11)\end{array}$ & \\
\hline \multicolumn{5}{|l|}{ Number of PPTFs, n (\%) } \\
\hline 0 & $72(43.4)$ & $31(43.7)$ & $41(43.2)$ & 0.4886 \\
\hline 1 & $28(16.9)$ & $14(19.7)$ & $14(14.7)$ & \\
\hline 2 & $34(20.5)$ & $10(14.1)$ & $24(25.3)$ & \\
\hline 3 & $5(3.0)$ & $2(2.8)$ & $3(3.2)$ & \\
\hline 4 & $17(10.2)$ & $8(11.3)$ & $9(9.5)$ & \\
\hline$\geq 5$ & $10(6.0)$ & $6(8.5)$ & $4(4.2)$ & \\
\hline \multicolumn{5}{|l|}{$\begin{array}{l}\text { Number of classes of preventive therapy used } \\
\text { previously, } \mathrm{n}(\%)\end{array}$} \\
\hline 1 & $34(20.5)$ & $9(12.7)$ & $25(26.3)$ & \\
\hline 2 & $10(6.0)$ & $2(2.8)$ & $8(8.4)$ & \\
\hline 3 & $5(3.0)$ & $4(5.6)$ & $1(1.1)$ & \\
\hline$>3$ & $4(2.4)$ & $2(2.8)$ & $2(2.1)$ & \\
\hline \multicolumn{5}{|c|}{$\begin{array}{l}\text { BMI, body mass index; MHD, monthly headache/migraine days; MSMD, monthly acute migraine- } \\
\text { specific medication days; } n \text {, number of patients; } N \text {, total number of patients; NA, not available; PPTF, } \\
\text { previous preventive treatment failure; SD, standard deviation }\end{array}$} \\
\hline \multicolumn{5}{|c|}{$\begin{array}{l}\text { Percentages are based on the total number of subjects in the full analysis set. P-value is derived from } \\
\text { a Chi-square test or exact test for categorical variables and from t-test or Wilcoxon test for continuous } \\
\text { variables. BMI is calculated as follows: (body weight in } \mathrm{kg} \text { )/ (height in } \mathrm{m})^{2} \text {; percentages for BMI } \\
\text { categories are based on the total number of patients who had BMI data in the full analysis set. }\end{array}$} \\
\hline
\end{tabular}

The mean (SD) number of MHD over one month prior to enrolment in the overall population was 15.6 (8.54) days and, as expected, was significantly higher with CM (22.9 [6.35] days) than with EM (9.0 [3.05] days; $p<0.0001)$. The mean (SD) number of MSMD at baseline was 12.8 (10.0) days, and this difference was statistically significant $(p<0.0001)$ between the $\mathrm{CM}(18.6$ [8.55] days) and EM subgroups (7.4 [8.11] days).

Baseline demographics and characteristics of all subgroups are presented in Table S1. All patients started erenumab at the 70-mg dose. The number of patients on erenumab $70 \mathrm{mg}$ throughout the study 
was 98 (59.0\%) and the dose was escalated to $140 \mathrm{mg}$ in 68 (41.0\%) patients. Overall, 72 (43.4\%) patients had no PPTFs prior to receiving erenumab, whereas 94 (56.6\%) had $\geq 1$ PPTF.

No statistically significant differences were observed in the proportion of patients with CM and EM who had comorbidities (Fig. 1a). Comorbidities by subgroup are presented in Fig. S1. In total, 53 patients (31.9\%) received at least one previous preventive treatment (Fig. 1b). Antiepileptics (15.7\%), tricyclic antidepressants (10.2\%), $\beta$-blockers (8.4\%) and calcium channel blockers $(7.8 \%)$ were the most frequently received previous preventive treatments (>5\% of patients). In this study, $100(60.2 \%)$ patients received erenumab as monotherapy, whereas 66 (39.8\%) received erenumab as an add-on to a current preventive therapy (Fig. 1b). Tricyclic antidepressants (15.7\%), antiepileptics (13.3\%), $\beta$-blockers (8.4\%) and calcium channel blockers (7.2\%) were the most frequently received current preventive treatments $(>5 \%$ of patients). There was a statistically significant difference between the proportions of patients receiving erenumab as monotherapy or add-on therapy $(p=0.0466)$, with higher rates of PPTFs in the add-on subgroup.

\section{Monthly headache/migraine days}

In the overall population, the mean (SD) number of MHD decreased from 15.7 (8.45) at baseline to 7.3 (7.54) at Month 1. A further decrease was noted at Month 3 (4.6 [4.83]) and Month 6 (4.1 [4.28]). The corresponding mean changes from baseline were -8.2 (8.83) at Month 1, -11.0 (9.15) at Month 3 and -11.3 (8.90) at Month 6 (Fig. 2). A reduction in MHD was observed from baseline to Months 1, 3 and 6 in both $\mathrm{CM}$ and EM groups (Fig. 2).

At all time points, most patients in the overall population achieved at least a $50 \%$ reduction from baseline in $\mathrm{MHD}(80 \%-91 \%)$, and the proportion achieving the 75 to $<100 \%$ or $100 \%$ reduction categories was $39.1 \%$ and $5.7 \%$ at Month $1 ; 46.5 \%$ and $14.1 \%$ at Month 3; and 52.6\% and 9.5\% at Month 6, respectively (Fig. 3). The mean (SD) number of MHD at baseline was 16.9 (8.83) in the erenumab monotherapy subgroup and 13.9 (7.57) in the erenumab add-on therapy subgroup. A greater reduction in the number of MHD was observed with erenumab monotherapy compared with erenumab as add-on therapy at all assessed time points, but the differences were not statistically significant ( $p>0.05$ for all time points; Fig. S2). A similar trend in the reduction of mean MHD was observed in the subgroups of patients who were prescribed and remained on erenumab $70 \mathrm{mg}$ and with dose escalation to $140 \mathrm{mg}$, as well as those in the treatment naïve and PPTF subgroups (Fig. S2). At all time points, most patients in the subgroup categories of with/without dose escalation, monotherapy/add-on therapy and treatment naïve/PPTF achieved at least a 50\% reduction from baseline in MHD. As anticipated, at Month 1, a higher proportion of patients in the PPTF subgroup had a reduction from baseline in MHD by $<50 \%$ versus those who were treatment naïve; however, this proportion improved over time with erenumab treatment (Fig. S3).

\section{Monthly acute migraine-specific medication days}


The mean (SD) MSMD at baseline in the overall population was 12.8 (10.00) and decreased to 4.1 (5.57) at Month 1, 2.9 (4.23) at Month 3 and 1.8 (3.02) at Month 6. The mean monthly change from baseline in MSMD was -9.0 (8.07) at Month 1 and was reduced further to -9.7 (8.73) at Month 3 and -10.7 (8.95) at Month 6 (Fig. 4). Similar to that in the overall population, there was a decrease in mean (SD) MSMD from baseline to Month 1, Month 3 and Month 6 in both CM and EM groups (Fig. 4).

Similar to the reduction in MHD, most patients in the overall population and CM and EM groups achieved at least a 50\% reduction from baseline in MSMD at all time points, and more than half of the patients in the EM group achieved a 100\% reduction in MSMD at all time points (Fig. 5). In the subgroups of patients with and without dose escalation, the mean monthly change from baseline in MSMD at Month 1 was 10.8 (9.61) and -8.1 (6.98), respectively; for patients who were treatment naïve and who had PPTF, the means changes were -8.9 (7.74) and -9.1 (8.32), respectively; and for those receiving erenumab as monotherapy or add-on therapy, the mean changes were -10.3 (8.25) and -7.7 (7.77), respectively (Fig. S4). In the subgroup of patients escalated to the $140-\mathrm{mg}$ dose, a majority achieved at least a $50 \%$ reduction from baseline at all time points, and the proportion of patients achieving either a $75 \%$ to $<100 \%$ or $100 \%$ reduction in MSMD increased from $76.5 \%$ at Month 3 (before dose escalation) to $84.2 \%$ at Month 6. The corresponding results in patients who remained on the 70 -mg dose were $54.9 \%$ at Month 1 , $65.5 \%$ at Month 3 and $82.7 \%$ at Month 6 (Fig. S5). A majority of patients achieved at least a $50 \%$ reduction at all time points in the monotherapy and add-on therapy subgroups and in the treatment naïve and PPTF subgroups. More than $35 \%$ of patients in the treatment naïve and PPTF subgroups had a $100 \%$ reduction in MSMD at all time points. Among those in the add-on therapy subgroup, $64 \%$ had a $100 \%$ reduction in MSMD by Month 6, increasing from 47.6\% at Month 1 and $45 \%$ at Month 3.

\section{Medication overuse}

There was a decrease in the number of patients in the overall population with overuse of at least one medication for $\geq 15$ days per month from $49(29.5 \%)$ at baseline to $12(7.2 \%), 6(3.6 \%)$ and 0 at Months 1 , 3 and 6 , respectively. Non-steroidal anti-inflammatory drugs were the most frequently used medications in the overall population at baseline (29 [17.5\%]), which declined to $4(2.4 \%)$ and $1(0.6 \%)$ at Months 1 and 3 , respectively.

\section{Exposure and safety}

A total of 167 patients were included in the safety set (one patient who met the exclusion criteria was on medication and was excluded from the FAS but was included in the safety set). The mean (SD) treatment duration and on-treatment exposure were 5.6 (1.54) and 6.6 (1.54) months, respectively. Of the 167 patients, 34 (20.4\%) experienced at least one AE. The most commonly reported AEs (>1\%) were constipation (3\%), insomnia (2.4\%) and influenza (1.8\%) followed by falls, dizziness, arthralgia, stress and headache (1.2\% each; Table 2 ). A majority (76.5\%) of AEs were mild to moderate in severity. Two patients $(1.2 \%)$ had severe AEs: atypical pneumonia and spontaneous abortion in one $(0.6 \%)$ patient 
each. In the remaining $4.8 \%$ of patients, the AE severity was unknown. Five patients (3.0\%) experienced at least one $A E$ that was related to study treatment. All five patients experienced constipation (3 [1.8\%] experienced mild AEs and the severity was unknown in 2 [1.2\%]). Approximately $41 \%$ of patients (68 of 167) had a dose change (all of these were dose escalation from $70 \mathrm{mg}$ to $140 \mathrm{mg}$ upon the treating physician's decision) during the study, but only three patients (1.8\%) experienced AEs leading to dose adjustments/interruptions. No deaths were reported.

\section{Table 2}

Treatment-emergent AEs (>1\% of patients) by preferred term and maximum severity (safety set)

\begin{tabular}{|c|c|c|c|c|c|}
\hline Preferred term & Mild & Moderate & Severe & Missing & $\begin{array}{l}\text { Overall } \\
N=167\end{array}$ \\
\hline $\begin{array}{l}\text { Number of patients with at least } 1 \mathrm{AE}, n \\
(\%)\end{array}$ & $\begin{array}{l}20 \\
(12.0)\end{array}$ & $6(3.6)$ & $2(1.2)$ & $8(4.8)$ & $\begin{array}{l}34 \\
(20.4)\end{array}$ \\
\hline Constipation & $3(1.8)$ & 0 & 0 & $2(1.2)$ & $5(3.0)$ \\
\hline Insomnia & $4(2.4)$ & 0 & 0 & 0 & $4(2.4)$ \\
\hline Influenza & $2(1.2)$ & 0 & 0 & $1(0.6)$ & $3(1.8)$ \\
\hline Fall & $1(0.6)$ & $1(0.6)$ & 0 & 0 & $2(1.2)$ \\
\hline Arthralgia & 0 & $1(0.6)$ & 0 & $1(0.6)$ & $2(1.2)$ \\
\hline Headache & $2(1.2)$ & 0 & 0 & 0 & $2(1.2)$ \\
\hline Stress & $2(1.2)$ & 0 & 0 & 0 & $2(1.2)$ \\
\hline Dizziness & $1(0.6)$ & 0 & 0 & $1(0.6)$ & $2(1.2)$ \\
\hline \multicolumn{6}{|c|}{$\begin{array}{l}\text { AE, adverse event; } n \text {, number of patients; } N \text {, total number of patients } \\
\text { Percentages are based on the total number of patients in the safety set. } \\
\text { A patient with multiple AEs within a primary system organ class is counted only once in the total row. } \\
\text { A patient with multiple occurrences of an } A E \text { under one treatment is counted only once in this } A E \\
\text { category. }\end{array}$} \\
\hline
\end{tabular}

\section{Discussion}

To the best of our knowledge, this is the first real-world study evaluating the experience of erenumab in patients with $\mathrm{CM}$ or EM in the UAE. A higher proportion of patients prescribed erenumab in the UAE were female, in the younger age categories, and more likely to have EM than CM [20]. The overall age distribution was older in the CM subgroup compared with the EM group, however, the mean age at onset of migraine was similar in both groups (approximately 29 years).

This study comprised both treatment-naïve patients (43\%) and those who had $\geq 1$ PPTF (57\%). Most patients included in the study were prescribed erenumab as monotherapy; approximately $40 \%$ of patients 
received concomitant preventive therapy, implying that these patients represented a more difficult-to-treat population. For the overall population, a reduction in the number of MHD was observed at all assessed time points with a noticeable reduction at Month 1 and further reductions at Months 3 and 6 . A similar trend was observed in the evaluation of MSMD. These findings were consistent with the results on change in MMD and MSMD from baseline observed in the global clinical trials of erenumab [14-17], as were the greater reductions in MHD and MSMD from baseline observed in patients with $\mathrm{CM}$ versus those with EM.

Concordant changes from baseline in MHD and MSMD were observed across the other subgroups that were studied (with and without dose escalation, treatment naïve and PPTF, monotherapy and add-on therapy). Of note, a greater reduction in MHD was observed in patients initiating erenumab as monotherapy compared with those initiating erenumab as add-on therapy, although this difference was not statistically significant. Patients receiving erenumab as add-on therapy may comprise a more difficult-to-treat population, but a reduction in MHD and MSMD was still observed. Our results support the ability of erenumab to provide additional clinical benefits when administered concomitantly with other forms of preventive therapy.

In the overall population and across all subgroups, most patients achieved $\mathrm{a} \geq 50 \%$ reduction in MHD and MSMD at all assessed time points and a substantial proportion of patients achieved 75 to $<100 \%$ and $100 \%$ reductions in MHD and MSMD during the study. Notably, this pattern of response was consistent in the subgroup of patients with dose escalation to $140 \mathrm{mg}$. This is in line with the erenumab prescribing information, which states that some patients can benefit from a dose increase from $70 \mathrm{mg}$ to 140 $\mathrm{mg}$ [12]. Similar to those observed in global clinical studies, reductions from baseline in MHD and MSMD were observed in both the treatment naïve and PPTF subgroups, with most patients in these subgroups achieving a $\geq 50 \%$ reduction from baseline in MHD and MSMD [21, 22]. However, there was a significant difference at Month 1 in the proportion of patients achieving a $\geq 50 \%$ reduction from baseline in MHD in the treatment naïve versus PPTF subgroups.

The safety profile reported here is similar to that reported in previous studies and no new safety signals were reported. Most AEs were mild to moderate in nature. Constipation was the most frequently reported AE. Interestingly, the overall proportion of patients reporting any $A E(20 \%)$ was lower in this study compared with that in global phase 2 and 3 studies (44\%-57\%) that evaluated erenumab $70 \mathrm{mg}$ or 140 $\mathrm{mg}$ for 3 or 6 months in patients with CM and/or EM [14-17, 23]. There were no discontinuations in this study, a finding that supports the favourable tolerability of erenumab.

The major limitations of this study are similar to those of any retrospectively designed studies, such as recall bias. By requiring patients to have taken at least 3 doses of erenumab for inclusion, the study may have excluded patients who discontinued treatment due to lack of efficacy or other reasons and may have skewed the results favourably in comparison to the pivotal clinical trial data. Headache is only one of the symptoms of migraine, and the selection of MHD instead of MMD as a secondary endpoint could be another limitation of this study. Additionally, headache and migraine days were not assessed 
separately, and headache type was abstracted only from information available in patient charts, which may lack granularity or consistency across centres [18].

\section{Conclusions}

In this real-world study of the use of erenumab in the UAE, patients who were prescribed erenumab were more likely to have EM than CM. Most patients received erenumab as monotherapy and approximately $57 \%$ had $\geq 1$ PPTF.

Irrespective of the demographics or baseline disease burden, clinically meaningful reductions in MHD and MSMD were observed at all assessed time points. In line with the higher baseline disease burden, the reduction in MHD and MSMD was greater in CM than EM. An additional clinical benefit was observed when erenumab was prescribed as add-on therapy and when its dose was increased from $70 \mathrm{mg}$ to 140 $\mathrm{mg}$ by physicians. This is in line with the consistent numerical advantage of the 140-mg dose over the 70mg dose observed on a range of endpoints in erenumab phase $2 / 3$ trials [24].

Overall, the results presented here from the UAE are consistent with the data generated in global clinical trial programmes [14-17]. No new safety events were observed. The AEs observed in this study were consistent with the established safety profile of erenumab. These real-world results from the UAE are representative of the patient population with migraine, which includes both treatment-naïve patients and those who had PPTFs; therefore, these findings could be applied to routine clinical practice.

These real-world data further extend the evidence on the clinically meaningful effectiveness of erenumab to the population of patients living with migraine in the UAE.

\section{Abbreviations}

AE Adverse event

BMI Body mass index

CGRP Calcitonin gene-related peptide

$\mathrm{CM}$ Chronic migraine

EM Episodic migraine

FAS Full analysis set

ICF Informed consent form

IRB Institutional Review Board

MHD Monthly headache/migraine days 
MMD Monthly migraine days

$\mathrm{MOH}$ Medication overuse headache

MSMD Migraine-specific medication days

PPTF Previous preventive treatment failure

SD Standard deviation

UAE United Arab Emirates

YLD Years lived with disability

\section{Declarations}

\section{Ethics approval and consent to participate}

All relevant ethical, health authority, data privacy and site-specific approvals were obtained prior to the start of the study. The study was conducted in accordance with the principles of the Declaration of Helsinki. Informed consent was obtained from patients before data collection. Depending on each site's local Institutional Review Board (IRB) regulations, a waiver of informed consent from the corresponding ethics committee was obtained. All authors were given access to the study data.

The study protocol and ICF consent waiver has been reviewed and approval granted by the below mentioned ethics committees and $\mathrm{HA}$ :

ACPN Institutional Review Board for ACPN site

Ministry of Health and Prevention for University of Sharjah site

University of Sharjah Ethics and Research Committee for University of Sharjah site

Dubai Scientific Research Ethics Committee for Rashid hospital site

\section{Consent for publication}

Not applicable

\section{Availability of data and materials}

The data that support the findings of this study are available from [Novartis Middle East FZE] but restrictions apply to the availability of these data, which were used under license for the current study, 
and so are not publicly available. Data are however available from the authors upon reasonable request and with permission of [Novartis Middle East FZE]

\section{Competing interests}

TA, SN and ABA have nothing to disclose. RV and SY are employees of Novartis Middle East FZE, Representative Office, Dubai, United Arab Emirates.

\section{Funding}

Novartis Middle East FZE, Representative Office, Dubai, United Arab Emirates funded the study design, data analysis and writing of the manuscript. Erenumab was co-developed by Amgen and Novartis.

\section{Authors' contributions}

All authors have reviewed and approved the final draft. All named authors meet the International Committee of Medical Journal Editors criteria for the authorship of this article, have contributed sufficiently to the work and provided critical revision of the manuscript for important intellectual content. All authors had full access to all data in this study and take complete responsibility for data integrity and the accuracy of data analysis. All authors have given their approval for this version to be published in BMC Neurology.

\section{Acknowledgements}

We thank Shyam Tiwari, Novartis Healthcare Pvt. Ltd., Hyderabad, India, for his contributions to this study; Swetha Sirimalla, Novartis Healthcare Pvt. Ltd., Hyderabad, India; and Paul Coyle, Novartis Ireland Ltd, Dublin, Ireland, for providing medical writing support, which encompassed writing of the manuscript, formatting, referencing, preparation of tables and figures as per journal guidelines and incorporating the authors' revisions and finalizing the draft for submission, all under the direction of the authors. All authors edited the manuscript for intellectual content, provided guidance during manuscript development and approved the final version submitted for publication.

\section{References}

1. Agosti R. Migraine burden of disease: From the patient's experience to a socio-economic view. Headache. 2018;58 Suppl 1:17-32; doi: 10.1111/head.13301.

2. Foster SA, Chen CC, Ding Y, Mason O, McGuiness CB, Morrow P, et al. Economic burden and risk factors of migraine disease progression in the US: a retrospective analysis of a commercial payer database. J Med Econ. 2020;23:1356-64; doi: 10.1080/13696998.2020.1814790. 
3. Collaborators GBDH. Global, regional, and national burden of migraine and tension-type headache, 1990-2016: a systematic analysis for the Global Burden of Disease Study 2016. Lancet Neurol. 2018;17:954-76; doi: 10.1016/S1474-4422(18)30322-3.

4. Steiner TJ, Stovner LJ, Jensen R, Uluduz D, Katsarava Z, Lifting The Burden: the Global Campaign against $\mathrm{H}$. Migraine remains second among the world's causes of disability, and first among young women: findings from GBD2019. J Headache Pain. 2020;21:137; doi: 10.1186/s10194-020-01208-0.

5. Benamer HT, Deleu D, Grosset D. Epidemiology of headache in Arab countries. J Headache Pain. 2010;11:1-3; doi: 10.1007/s10194-009-0173-8.

6. Al-Hashel JY, Ahmed SF, Alroughani R. Burden of migraine in a Kuwaiti population: a door-to-door survey. J Headache Pain. 2017;18 1:105; doi: 10.1186/s10194-017-0814-2.

7. Hepp Z, Bloudek LM, Varon SF. Systematic review of migraine prophylaxis adherence and persistence. J Manag Care Pharm. 2014;20:22-33; doi: 10.18553/jmcp.2014.20.1.22.

8. Lassen LH, Haderslev PA, Jacobsen VB, Iversen HK, Sperling B, Olesen J. CGRP may play a causative role in migraine. Cephalalgia. 2002;22:54-61; doi: 10.1046/j.1468-2982.2002.00310.x.

9. Chiang CC, Schwedt TJ. Calcitonin gene-related peptide (CGRP)-targeted therapies as preventive and acute treatments for migraine-The monoclonal antibodies and gepants. Prog Brain Res. 2020;255:143-70; doi: 10.1016/bs.pbr.2020.06.019.

10. Peters GL. Migraine overview and summary of current and emerging treatment options. Am J Manag Care. 2019;25:S23-S34.

11. King CT, Gegg CV, Hu SN, Sen Lu H, Chan BM, Berry KA, et al. Discovery of the Migraine Prevention Therapeutic Aimovig (Erenumab), the First FDA-Approved Antibody against a G-Protein-Coupled Receptor. ACS Pharmacol TransI Sci. 2019;2:485-90; doi: 10.1021/acsptsci.9b00061.

12. Aimovig® (erenumab-aooe) [prescribing Information]. Amgen Inc., Thousand Oaks, CA. April 2020.https://www.accessdata.fda.gov/drugsatfda_docs/label/2018/761077s000lbl.pdf. Accessed June 252021.

13. Aimovig ${ }^{\circledR}$ (erenumab-aooe) [summary of product characteristics]. Amgen Inc., Thousand Oaks, CA. April 2020.https://www.ema.europa.eu/en/documents/product-information/aimovig-epar-productinformation_en.pdf. Accessed June 252021.

14. Goadsby PJ, Reuter U, Hallstrom Y, Broessner G, Bonner JH, Zhang F, et al. A controlled trial of erenumab for episodic migraine. N Engl J Med. 2017;377:2123-32; doi: 10.1056/NEJMoa1705848.

15. Reuter U, Goadsby PJ, Lanteri-Minet M, Wen S, Hours-Zesiger P, Ferrari MD, et al. Efficacy and tolerability of erenumab in patients with episodic migraine in whom two-to-four previous preventive treatments were unsuccessful: a randomised, double-blind, placebo-controlled, phase $3 \mathrm{~b}$ study. Lancet. 2018;392:2280-7; doi: 10.1016/S0140-6736(18)32534-0.

16. Sun H, Dodick DW, Silberstein S, Goadsby PJ, Reuter U, Ashina M, et al. Safety and efficacy of AMG 334 for prevention of episodic migraine: a randomised, double-blind, placebo-controlled, phase 2 trial. Lancet Neurol. 2016;15:382-90; doi: 10.1016/S1474-4422(16)00019-3. 
17. Tepper S, Ashina M, Reuter U, Brandes JL, Dolezil D, Silberstein S, et al. Safety and efficacy of erenumab for preventive treatment of chronic migraine: a randomised, double-blind, placebocontrolled phase 2 trial. Lancet Neurol. 2017;16:425-34; doi: 10.1016/S1474-4422(17)30083-2.

18. Faust E, Pivneva I, Yang K, Betts KA, Ahmed Z, Joshi S, et al. Real-World Treatment Profiles, Clinical Outcomes, and Healthcare Resource Utilization of Patients with Migraine Prescribed Erenumab: A Multicenter Chart-Review Study of US Headache Centers. Neurol Ther. 2021;10 1:293-306; doi: 10.1007/s40120-021-00245-4.

19. Headache Classification Committee of the International Headache S. The International Classification of Headache Disorders, 3rd edition (beta version). Cephalalgia. 2013;33:629-808; doi: $10.1177 / 0333102413485658$.

20. Ashina M, Katsarava Z, Do TP, Buse DC, Pozo-Rosich P, Ozge A, et al. Migraine: epidemiology and systems of care. Lancet. 2021;397 10283:1485-95; doi: 10.1016/S0140-6736(20)32160-7.

21. Ashina M, Tepper S, Brandes JL, Reuter U, Boudreau G, Dolezil D, et al. Efficacy and safety of erenumab (AMG334) in chronic migraine patients with prior preventive treatment failure: A subgroup analysis of a randomized, double-blind, placebo-controlled study. Cephalalgia. 2018;38:1611-21; doi: $10.1177 / 0333102418788347$.

22. Goadsby PJ, Paemeleire K, Broessner G, Brandes J, Klatt J, Zhang F, et al. Efficacy and safety of erenumab (AMG334) in episodic migraine patients with prior preventive treatment failure: $A$ subgroup analysis of a randomized, double-blind, placebo-controlled study. Cephalalgia. 2019;39:817-26; doi: 10.1177/0333102419835459.

23. Andreou AP, Fuccaro M, Lambru G. The role of erenumab in the treatment of migraine. Ther Adv Neurol Disord. 2020;13:1756286420927119; doi: 10.1177/1756286420927119.

24. Goadsby PJ, Reuter U, Hallstrom Y, Broessner G, Bonner JH, Zhang F, et al. One-year sustained efficacy of erenumab in episodic migraine: Results of the STRIVE study. Neurology. 2020;95:e469e79; doi: 10.1212/WNL.0000000000010019.

\section{Figures}




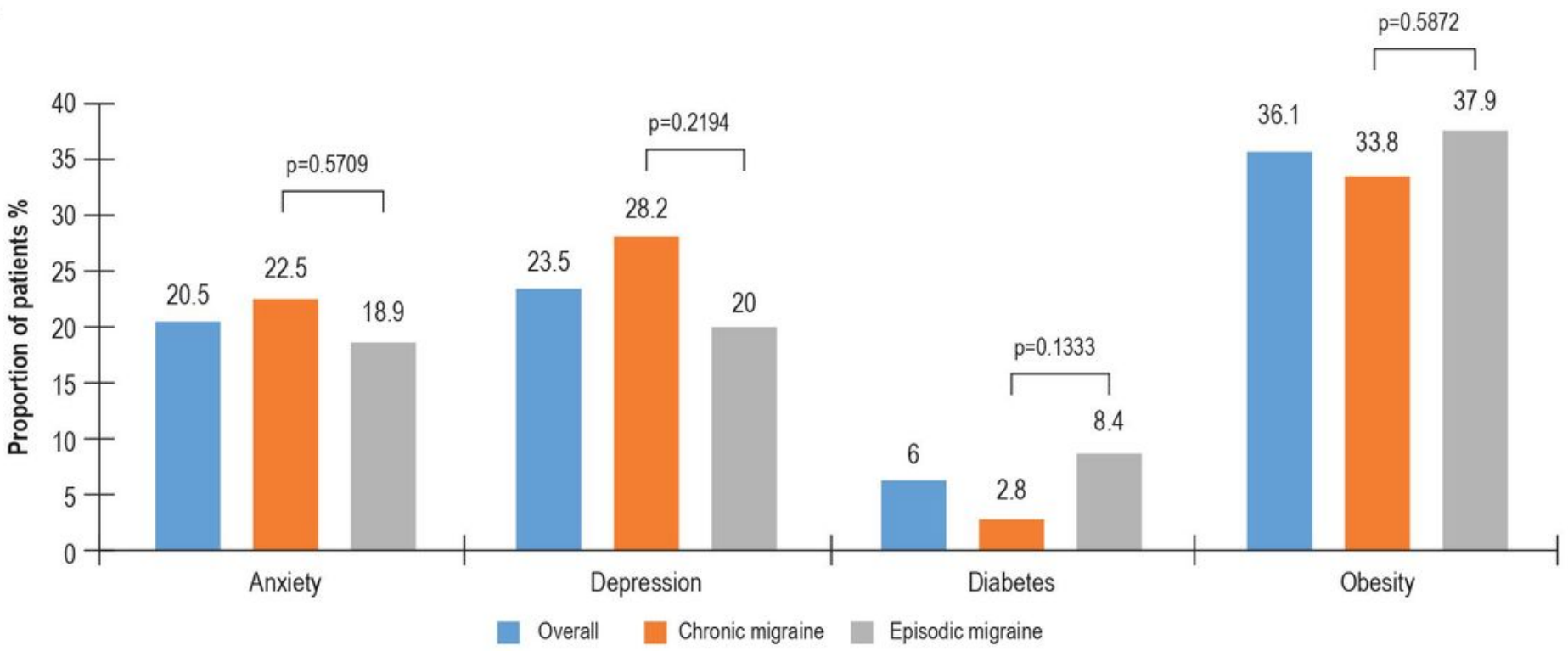

b

\section{Previous preventive treatment}

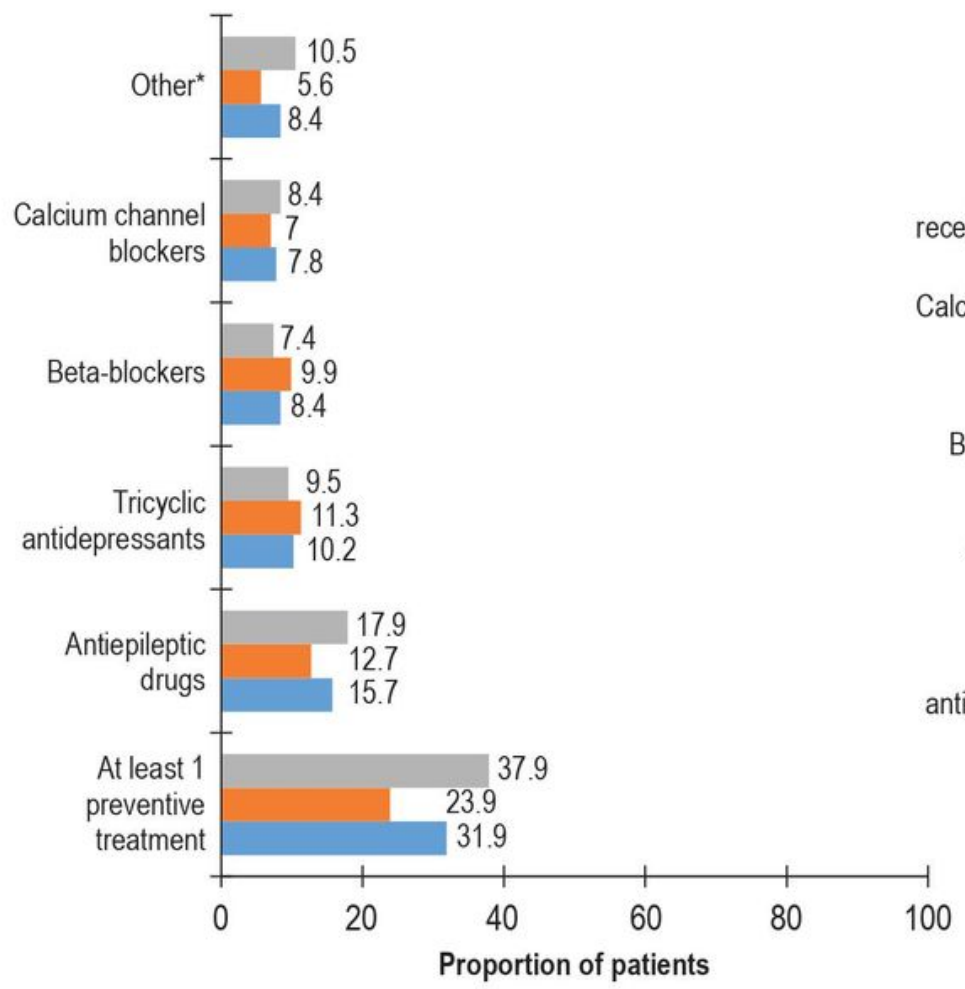

Episodic migraine

\section{Current preventive treatment}

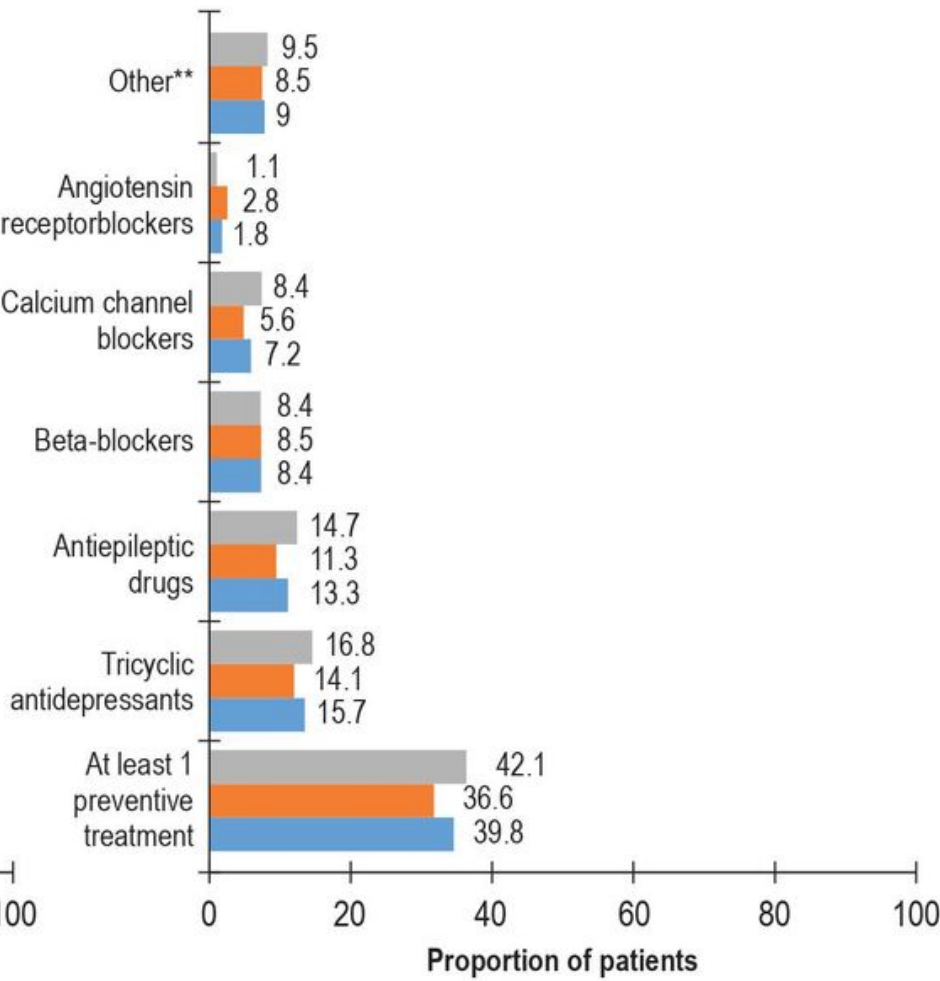

Chronic migraine

Overall

\section{Figure 1}

a Proportion of patients with comorbidities - by overall population, migraine type and monotherapy and add-on therapy

b Proportion of patients with previous or current preventive treatment - Overall population and by migraine type 
*Botulinum toxin type A, antiepileptics, antimigraine preparations, flunarizine dihydrochloride, galcanezumab, local anaesthetics.

**Magnesium oxide, botulinum toxin type A, antiepileptics, antimigraine preparations, local anaesthetics, magnesium, venlafaxine

Percentages are based on the total number of subjects in the full analysis set.
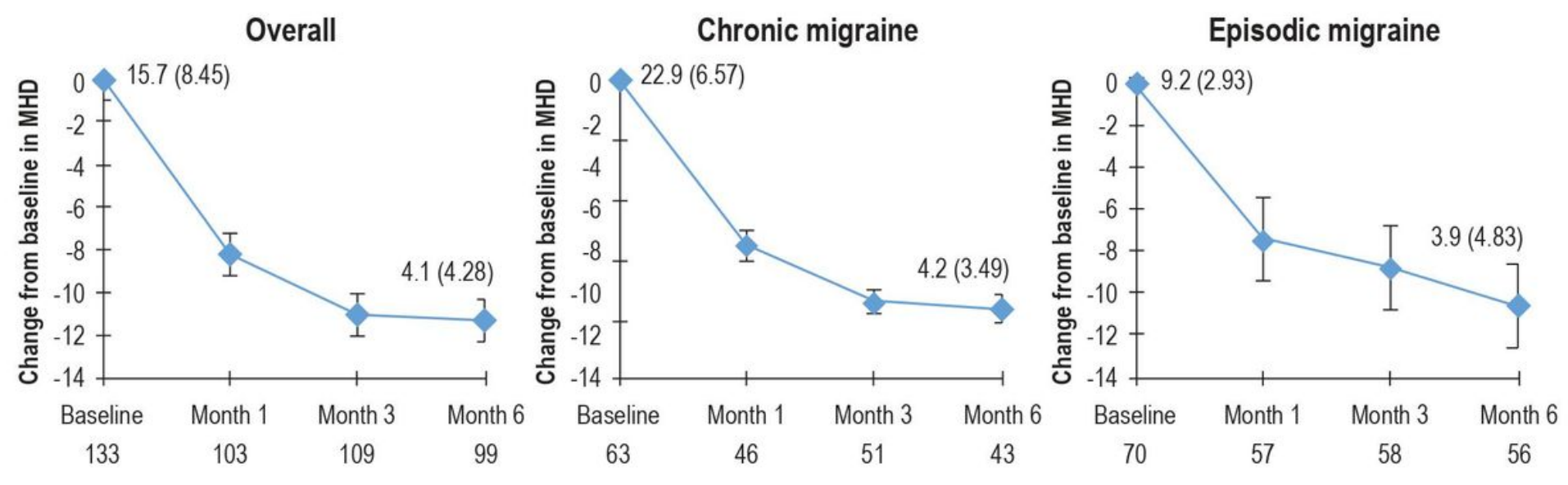

\section{Figure 2}

Change from baseline in MHD - Overall population and by migraine type

MHD, monthly headache/migraine days; SD, standard deviation

Mean \pm standard error values are plotted and baseline MHD (SD) are provided for each plot.

The total number of evaluable subjects at the respective visit in the full analysis set are presented below each plot.

Baseline is defined as the last observation on the day of or before the first dose of the study drug.

After baseline, only patients with a value at both baseline and the respective month are included. 


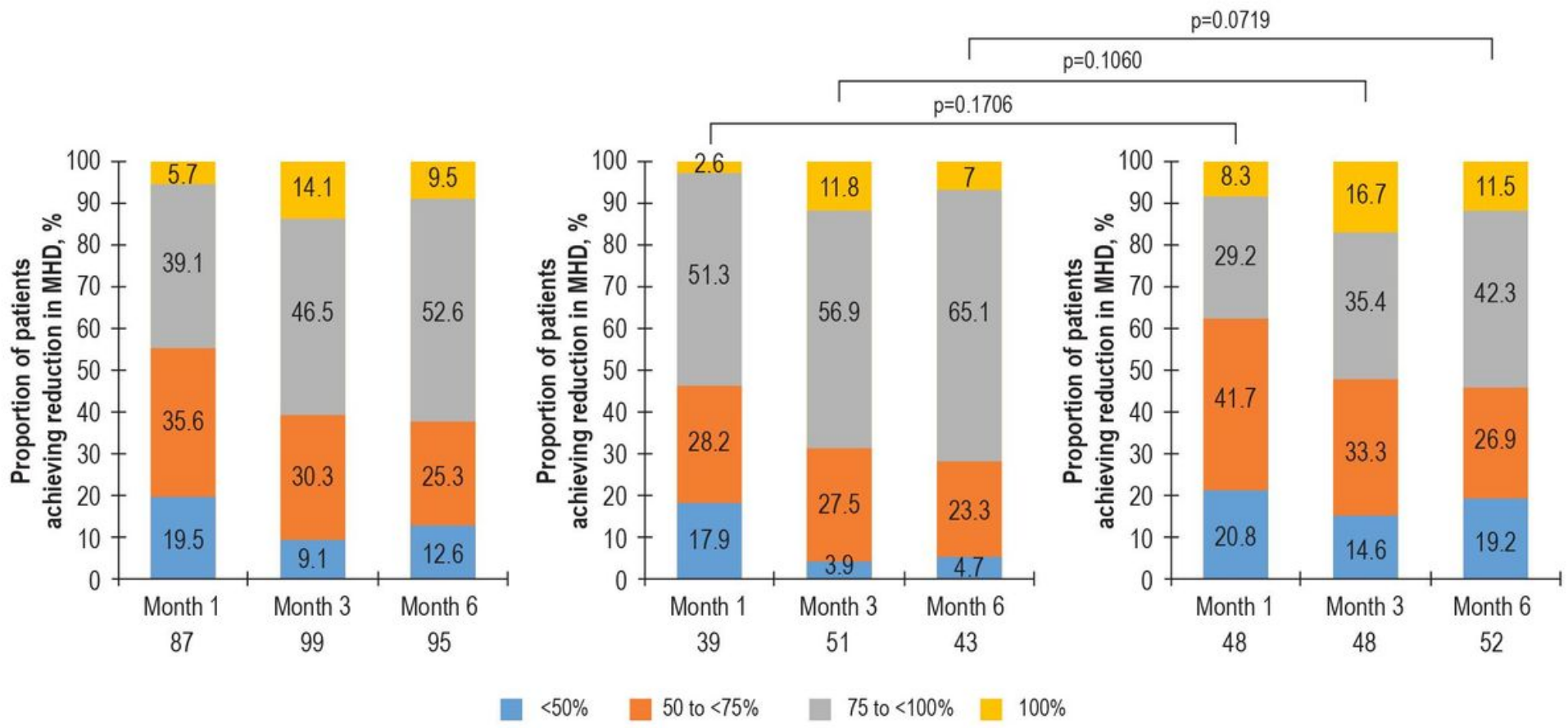

\section{Figure 3}

Proportion of patients achieving $<50 \%, 50 \%$ to $<75 \%, 75 \%$ to $<100 \%$ and $100 \%$ reduction in MHD over time MHD, monthly Percentages are based on the total number of evaluable subjects at the respective visit in the full analysis set.

Percentages are based on the total number of evaluable subjects at the respective visit in the full analysis set.
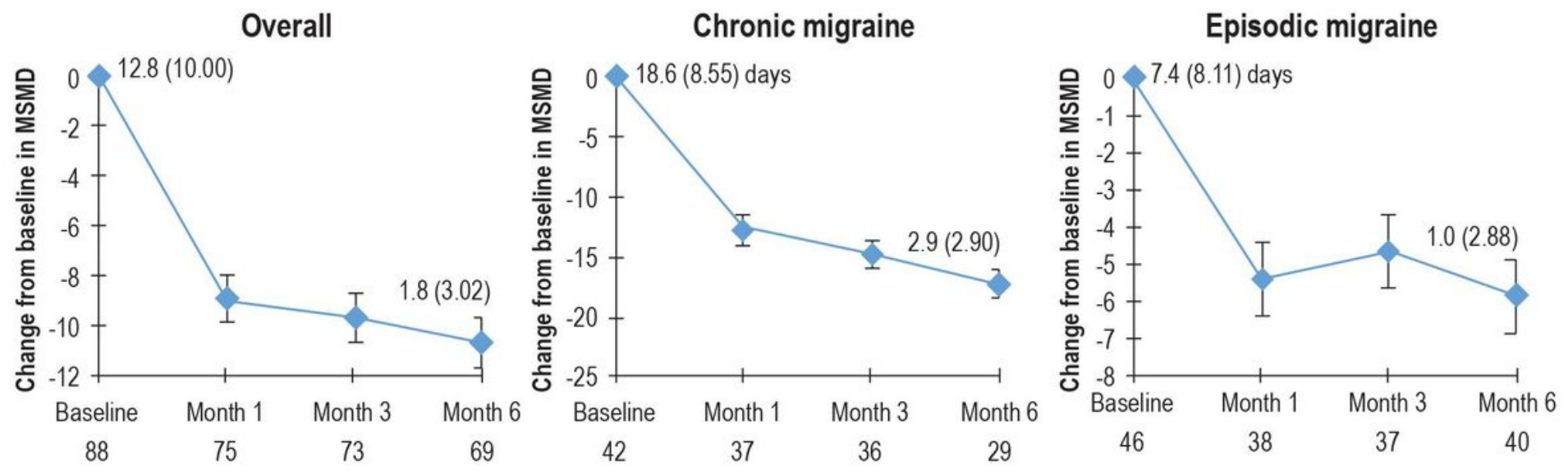

\section{Figure 4}

Change from baseline in MSMD - Overall population and by migraine type

MSMD, monthly acute migraine-specific medication days; SD, standard deviation 
Mean \pm standard error values are plotted and baseline MSMD (SD) are provided for each plot.

Baseline is defined as the last observation on the day of or before the first dose of the study drug.

After baseline, only patients with a value at both baseline and the respective month are included.

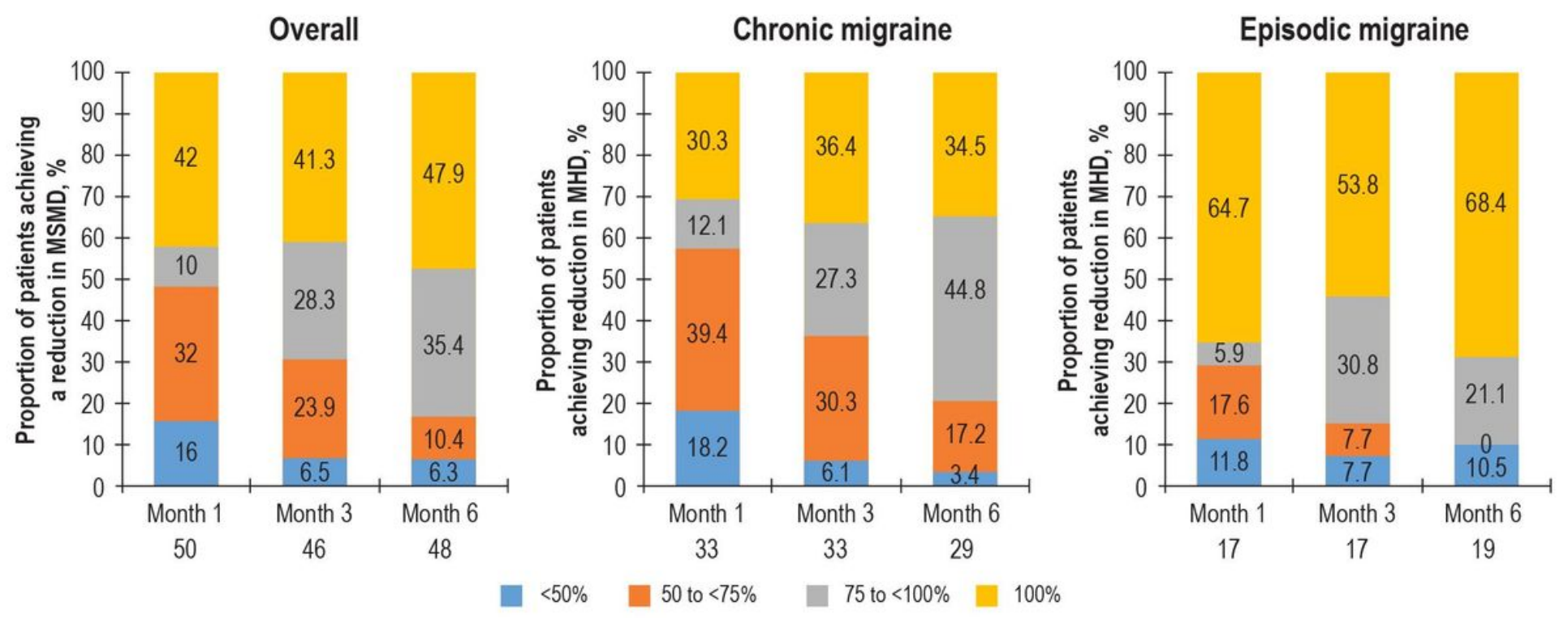

Figure 5

Proportion of patients achieving $<50 \%, 50 \%$ to $<75 \%, 75 \%$ to $<100 \%$ and $100 \%$ reduction in MSMD

MSMD, monthly acute migraine-specific medication days

Percentages are based on the total number of evaluable patients at the respective visit in the full analysis set.

\section{Supplementary Files}

This is a list of supplementary files associated with this preprint. Click to download.

- Additionalfile1supplfileErenumabinMigraineinUAEJan252022.docx

- additionalfile2.pdf 